\title{
Simulating Arbitrary Pair-Interactions by a Given Hamiltonian: Graph-Theoretical Bounds on the Time Complexity
}

\author{
P. Wocjan, D. Janzing, and Th. Beth \\ Institut für Algorithmen und Kognitive Systeme, \\ Universität Karlsruhe \\ Am Fasanengarten 5, D-76 131 Karlsruhe, Germany \\ June 13, 2001
}

\begin{abstract}
We use an $n$-spin system with permutation symmetric $z z$-interaction for simulating arbitrary pair-interaction Hamiltonians. The calculation of the required time overhead is mathematically equivalent to a separability problem of $n$-qubit density matrices. We derive lower and upper bounds in terms of chromatic index and the spectrum of the interaction graph. The complexity measure defined by such a computational model is related to gate complexity and a continuous complexity measure introduced in a former paper. We use majorization of graph spectra for classifying Hamiltonians with respect to their computational power.
\end{abstract}

\section{Introduction}

The most common models for quantum computers use single and two qubit gates as basic transformations in order to generate arbitrary unitary operations on the quantum registers. Most discussions about the generation of quantum algorithms, quantum codes and possible realizations had successfully been based on this concept. Mostly, even the definition of quantum complexity refers to such a model [1]. Nevertheless there is a priori no reason, why two qubit gates should be considered as basic operations for future quantum computers. In principle every quantum system could serve as a quantum register provided that its time evolution can be controlled in a universal way. At first sight, every definition of quantum complexity seems hence to be adequate only for a specific model of quantum computation. But it seems to be a rather general feature of Hamiltonians available in nature that particles interact with other particles in such a form, that the total Hamiltonian is a sum of pair-interactions. Therefore we want to base quantum complexity theory only on such a general feature. This

\footnotetext{
${ }^{1}$ This might be seen in the spirit of D. Deutsch's statement "What computers can or cannot compute is determined by the laws of physics alone and not by pure mathematics." [1], Chapter II
} 
feature justifies the following control theoretic model: If $n$ qubits are assumed to be physically represented by $n$ particles, the only part of the system's Hamiltonian which can be changed by extern access is the free Hamiltonian of each qubit. These 1-particle Hamiltonians might be controllable since they are only effective Hamiltonians which are phenomenologically given by an interaction to many extern particles (mean-field approximation [2]). Based on results of quantum control theory in 2-spin systems [3] we investigate the problem of simulating arbitrary pair-interaction Hamiltonians by a given one. We assume that the Hamiltonian of the $n$-system is a permutation invariant $z z$-interaction and show that the computational power? of this Hamiltonian (together with local transformations on each spin) is at least as large as the power of quantum computers with 2 qubit gates. For infinitesimal time evolutions, it turns out to be even stronger.

We develop a theory, where the computational power of a Hamiltonian for simulating arbitrary Hamiltonians is characterized by features of the interactions graphs. Standard concepts of graph theory like chromatic index and spectrum of the adjacency matrix together with majorization turn out to provide lower and upper bounds on the simulation overhead. Here we are interested in the exact overhead and not only in polynomial equivalence as in [4].

\section{Our model of computation}

Based on the approach of [3] we consider the following model. The quantum system is a spin system, i.e. its Hilbert space is $\mathcal{H}_{n}=\left(\mathbb{C}^{2}\right)^{\otimes n}$, and its Hamiltonian $H_{d} \in \mathfrak{s u}\left(2^{n}\right)$ consists only of pair-interactions, i.e.

$$
H_{d}=\sum_{1 \leq k<l \leq n} H_{k, l}
$$

where $H_{k l}$ acts only on the Hilbert space of the qubits $k$ and $l$. We assume that for every $k$ and $l$ the Hamiltonian $H_{k l}$ describes a non-trivial coupling and is traceless. The system's Hamiltonian $H_{d}$ is also called the drift Hamiltonian since it is always present. We assume that we can perform all unitaries in the control group $K=$ $S U(2) \otimes \cdots \otimes S U(2)$ arbitrarily fast compared to the time evolution of the internal couplings between the qubits. Let $G$ be the unitary Lie group $S U\left(2^{n}\right)$ and $u \in G$ be a unitary we want to realize. To achieve this all we can do is perform $v_{1} \in K$, wait $t_{1}$, perform $v_{2} \in K$, wait $t_{2}, \ldots$, perform $v_{p} \in K$ and wait $t_{p}$. The resulting unitary is

$$
u=\exp \left(i H_{d} t_{p}\right) v_{p} \cdots \exp \left(i H_{d} t_{2}\right) v_{2} \exp \left(i H_{d} t_{1}\right) v_{1} .
$$

This can be written as

$$
u=k_{p} \exp \left(i k_{p}^{\dagger} H_{d} k_{p}\right) \cdots \exp \left(i k_{2}^{\dagger} H_{d} k_{2}\right) \exp \left(i k_{1}^{\dagger} H_{d} k_{1}\right)
$$

where $k_{i}=v_{i} \cdots v_{1}$ for $i=1, \ldots, p$. This is just the solution of a time-dependent Schrödinger equation with piecewise constant Hamiltonians - conjugates of the drift

\footnotetext{
${ }^{2}$ in the sense of time required to generate unitaries
} 
Hamiltonian $H_{d}$ by unitaries of $K$ - followed by the unitary $k_{p} \in K$. Let $A d_{K}\left(H_{d}\right)$ denote the conjugacy class

$$
A d_{K}\left(H_{d}\right)=\left\{A d_{k}\left(H_{d}\right)=k^{\dagger} H_{d} k \mid k \in K\right\} .
$$

Definition 1 A continuous time algorithm A of running time $T$ is a piecewise constant function $t \mapsto H(t)$ from the interval $[0, T]$ onto the set $A d_{K}\left(H_{d}\right)$ followed by some local unitary $k \in K$. We say $A$ implements $u$ if $u=k u(T)$ where $(u(t))_{t \in[0, T]}$ is the solution of the time-dependent Schrödinger equation $(d / d t) u(t)=-i H(t) u(t)$ with $u(0)=I$.

The complexity of a unitary in this model is the running time of the optimal continuous time algorithms.

Let $\sigma_{\alpha}^{i}$ denote the Pauli spin matrix $\sigma_{\alpha}(\alpha=x, y, z)$ that acts on the $i$ th spin. For simplicity, we assume that the drift Hamiltonian is

$$
H_{d}=\sum_{1 \leq k<l \leq n} \sigma_{z}^{k} \sigma_{z}^{l}
$$

The physical systems we have in mind might be for example solid states with longrange interactions. Of course one might object that the interaction strength always decreases with the distance between the interacting particles. It will turn out that the assumption on non-decreasing interaction strengths makes our model rather strong with respect to its computational power. One should understand our assumptions as the attempt to use a strong computational model which is still physically justificable. Many aspects of our theory can be developed in strong analogy for more general drift Hamiltonians.

In Section 3 we will compare the computational power of our model with the power of quantum computers based on 2-qubit gates. Our arguments refer always to infinitesimal time evolutions, i.e., we will show that our model can implement quantum gates without overhead since we can simulate the time evolution implementing parallelized quantum gates.

First we have to define what we mean by simulating the time evolution $\exp (i H t)$ during a small time interval $[0, \epsilon]$ where $H$ is an arbitrary pair-interaction Hamiltonian. Assume we have written $H$ as a positive linear combination $H=\sum_{j} \mu_{j} H_{j}$ with $\mu_{j}>0$ and each $H_{j}$ is an element of the conjugacy class $A d_{K}\left(H_{d}\right)$. For small $\epsilon$ the unitary

$$
\prod_{j} \exp \left(i \epsilon \mu_{j} H_{j}\right)
$$

is a good approximation for

$$
\exp (i \epsilon H)=\exp \left(i \epsilon \sum_{j} \mu_{j} H_{j}\right)
$$

This approximation is implemented if the system evolves the time $\epsilon \mu_{j}$ with respect to the Hamiltonian $H_{j}$. The sum $\mu=\sum_{j} \mu_{j}$ is exactly the time overhead of the simulation. Hence the problem is to express $H$ as a positive linear combination such that the overhead $\mu$ is minimal. Of course such a procedure might not be optimal if one 
were interested in the implementation of $\exp (i H s)$ for any special value of $s$. Here we want to imitate the whole dynamical time evolution $(\exp (i H s))_{s>0}$ in arbitrary small steps $\epsilon$. Then the optimization reduces clearly to the convex problem stated above.

In the following it will be convenient to use a concise representation for the drift Hamiltonian and the interaction to be simulated: a pair interaction Hamiltonian between qubits $k$ and $l$ can be written as

$$
H_{k l}=\sum_{\alpha, \beta=x, y, z} J_{k l ; \alpha \beta} \sigma_{\alpha}^{k} \sigma_{\beta}^{l} .
$$

The strengths of the components are represented by the pair-interaction matrix

$$
J_{k l}=\left(\begin{array}{ccc}
J_{k l ; x x} & J_{k l ; x y} & J_{k l ; x z} \\
J_{k l ; y x} & J_{k l ; y y} & J_{k l ; y z} \\
J_{k l ; z x} & J_{k l ; z y} & J_{k l ; z z}
\end{array}\right) \in \mathbb{R}^{3 \times 3} .
$$

The total Hamiltonian $H$ is represented by the $J$-matrix

$$
J=\left(\begin{array}{c|c|c|c|c}
0 & J_{12} & J_{13} & \cdots & J_{1 n} \\
\hline J_{21} & 0 & J_{23} & \cdots & J_{2 n} \\
\hline J_{31} & J_{32} & 0 & & J_{3 n} \\
\hline \vdots & \vdots & & \ddots & \\
\hline J_{n 1} & J_{n 2} & J_{n 3} & & 0
\end{array}\right) \in \mathbb{R}^{3 n \times 3 n}
$$

To explain more explicitly, why our simulation problem is a convex optimization, we recall that every convex combination $\mu H_{1}+(1-\mu) H_{2}$ of two Hamiltonians $H_{1}$ and $H_{2}$ can be simulated with overhead 1 if $H_{1}$ and $H_{2}$ can. Remarkably, the problem of specifying the set of Hamiltonians which can be simulated with overhead 1 is related to the problem of generalizing Bell inequalities to $n$-qubit states. More specifically, the convex problem can be reduced to the question 'how strong can 2-spin correlations be in a separable $n$-qubit quantum state?'

Theorem 1 (Optimal simulation) The Hamiltonian $H$ can be simulated with overhead $\mu$ if and only if there is a separable quantum state $\rho$ in $\left(\mathbb{C}^{2}\right)^{\otimes n}$ such that

$$
\frac{1}{\mu} J+I=\left(\operatorname{tr}\left(\rho \sigma_{\alpha}^{k} \sigma_{\beta}^{l}\right)\right)_{k l ; \alpha \beta}
$$

where $J$ denotes the $J$-matrix of $H$ and $I$ the $3 n \times 3 n$ identity matrix.

Proof: By rescaling the considered Hamiltonian, it is sufficient to show that this is true for all Hamiltonians in $A d_{K}\left(H_{d}\right)$ with $\mu=1$. Assume we have written $H$ as a convex combination $H=\sum_{j} \mu_{j} H_{j}$ with $H_{j} \in A d_{K}\left(H_{d}\right)$. In order to show that there is a separable state of the desired form it is sufficient to show that the $J$-matrix of each Hamiltonian $H_{j}$ satisfies the equation of the theorem for an appropriate separable state. Let $H_{j}=u H_{d} u^{\dagger}$. $H_{j}$ can be represented by $n$ three dimensional real unit vectors: to each qubit we associate the vector $\left|J_{k}\right\rangle=\left(J_{k ; x}, J_{k ; y}, J_{k ; z}\right)^{t} \in \mathbb{R}^{3}$ where $u_{k} \sigma_{z} u_{k}^{\dagger}=J_{k ; x} \sigma_{x}+J_{k ; y} \sigma_{y}+J_{k ; z} \sigma_{z}$ and $u=u_{1} \otimes \ldots \otimes u_{n}$. The pair-interaction matrices are given by the matrix products $J_{k l}=\left|J_{k}\right\rangle\left\langle J_{l}\right|$. 
By the Bloch sphere representation we have a correspondence between the unit vectors $\left|J_{k}\right\rangle$ and the projections $\rho_{k}$ in $\mathbb{C}^{2}$ defined by $J_{k ; \alpha}=\operatorname{tr}\left(\rho_{k} \sigma_{\alpha}\right)$. Let $\rho$ be the product state $\rho:=\rho_{1} \otimes \ldots \otimes \rho_{n}$. Then we have $J_{k l ; \alpha \beta}=\operatorname{tr}\left(\rho \sigma_{\alpha}^{k} \sigma_{\beta}^{l}\right)$ for all $k \neq l$. Note that the product of two different Pauli matrices is the third Pauli matrix multiplied by a scalar. The only problem that remains is that we may have $\operatorname{tr}\left(\rho \sigma_{\alpha}^{k} \sigma_{\beta}^{k}\right) \neq 0$ for $\alpha \neq \beta$. We substitute $\rho$ by a state $\bar{\rho}$ in such a way that the expectation values of all traceless 1-qubit observables vanish and the expectation values of all considered 2-qubit observables remain unchanged. For every $\left|J_{k}\right\rangle$ we can find $U_{k}^{\prime} \in S O(3)$ such that $U_{k}^{\prime}\left|J_{k}\right\rangle=-\left|J_{k}\right\rangle$. This rotation corresponds to conjugation of the qubit $k$ by a unitary $u_{k}^{\prime}$. To $-\left|J_{k}\right\rangle$ corresponds the projection $\rho_{k}^{\prime}:=I_{2}-\rho_{k}$. Let

$$
\bar{\rho}:=\frac{1}{2}\left(\rho_{1} \otimes \ldots \otimes \rho_{n}+\rho_{1}^{\prime} \otimes \ldots \otimes \rho_{n}^{\prime}\right) .
$$

Then we have $\operatorname{tr}\left(\bar{\rho} \sigma_{\alpha}^{k} \sigma_{\beta}^{l}\right)=\operatorname{tr}\left(\rho \sigma_{\alpha}^{k} \sigma_{\beta}^{l}\right.$ ) for all $k \neq l$ (all vectors are multiplied by -1 and therefore there is no effect on the pairs) and $J_{k k}$ is the $3 \times 3$ identity matrix.

Assume conversely we have a separable state of the desired form. Take its decomposition into pure product states. By the Bloch sphere representation we obtain the required conjugations of the drift Hamiltonian $H_{d}$. The time they have to be applied are given by the coefficients in the convex decomposition.

\section{Lower and upper bounds}

A simple lower bound on the simulation time overhead can be derived from the fact that $J / \mu+I$ has to be a positive matrix, which is an easy conclusion from Theorem 1 .

Corollary 1 (Lower bound) The absolute value of the smallest eigenvalue of the $J$-matrix is a lower bound on the simulation overhead of $H$.

Proof: The matrix

$$
\left(\operatorname{tr}\left(\rho \sigma_{\alpha}^{k} \sigma_{\beta}^{l}\right)\right)_{k l ; \alpha \beta}
$$

is positive for every state $\rho$ in $\left(\mathbb{C}^{2}\right)^{\otimes n}$ : let $|d\rangle=\left(d_{k ; \alpha}\right)$ be an arbitrary vector and $A=\sum_{k, \alpha} d_{k ; \alpha} \sigma_{\alpha}^{k}$. Then we have

$$
\sum_{k, l, \alpha, \beta} d_{k ; \alpha} \operatorname{tr}\left(\rho \sigma_{\alpha}^{k} \sigma_{\beta}^{l}\right) d_{l ; \beta}=\operatorname{tr}\left(\rho A A^{*}\right) \geq 0 .
$$

Now we show that our computational model is at least as powerful as the usual model with 2-qubit gates, even if also cares about constant overhead. We describe here briefly the quantum circuit model and introduce the weighted depth following [11]. It is a complexity measure for unitary transformations based on the quantum circuit model. We assume that two qubit gates acting on disjoint pairs of qubits can be implemented simultaneously and define:

Definition $2 A$ quantum circuit $A$ of depth $k$ is a sequence of $s$ steps $\left\{A_{1}, \ldots, A_{s}\right\}$ where every step consists of a set of two qubit gates $\left\{u_{k l}\right\}_{k, l}$ acting on disjoint pairs $(k, l)$ of qubits. Every step $i$ defines a unitary operator $v_{i}$ by taking the product of all corresponding unitaries in any order. The product $u:=\Pi_{i \leq s} v_{i}$ is the 'unitary operator implemented by $A$ '. 
The following quantity measures the deviation of a unitary operator from the identity:

Definition 3 The angle of an arbitrary unitary operator $u \in S U(4)$ is the smallest possible norm $\|a\|$ of a self-adjoint operator $a \in \mathfrak{s u}(4)$ which satisfies $\exp (i a)=u$.

It coincides with the time required for the implementation of $u$ if the norm of the used Hamiltonian is 1 . We consider only the angle of two-qubit gates, i.e. we do not include the angle of local gates in the definition of the weighted depth. The notion of angle allows us to formulate a modification of the term 'depth' which will later turn out to be decisive in connecting complexity measures of discrete and continuous algorithms:

Definition 4 Let $\alpha_{i}$ be the maximal angle of the unitaries performed in step $i$. Then the weighted depth is defined to be the sum $\alpha=\sum_{i} \alpha_{i}$.

Assuming that the implementation time of a unitary is proportional to its angle, the weighted depth is the running time of the algorithm. We first need two technical lemmas to show that such an algorithm can be simulated by our computational model with complete $z z$-Hamiltonian without any time overhead.

Lemma 1 Let $M$ be a set of qubit pairs, such that no two pairs contain a common qubit. Then we can simulate

$$
H_{M}=\sum_{(k, l) \in M} \sigma_{z}^{k} \sigma_{z}^{l}
$$

with overhead 1.

Proof: This has been noted in [5]. Theorem 3 proves a more general statement.

Lemma 2 Let $H_{d}=\sigma_{z} \otimes \sigma_{z}$ be the drift Hamiltonian of a 2-spin system. All Hamiltonians $H \in \mathfrak{s u}(4)$ can be simulated with overhead less than $\|H\|$.

Proof: We first assume that $H$ contains no local terms, i.e. $H=\sum_{\alpha, \beta} J_{\alpha \beta} \sigma_{\alpha} \otimes \sigma_{\beta}$. Let $J_{12}$ be the matrix representing $H$. Conjugation of $H$ by $k=u \otimes v \in S U(2) \otimes S U(2)$ corresponds to multiplication of $J_{12}$ by $U \in S O(3)$ from the left and by $V \in S O(3)$ from the right. By the singular value decomposition [9] there are $U, V \in S O(3)$ such that $J_{12}=U \operatorname{diag}\left(s_{x}, s_{y}, s_{z}\right) V$ where $s_{x}, s_{y}, s_{z}$ are the singular values of $J_{12}$. Equivalently, there is $k \in S U(2) \otimes S U(2)$ such that $k H k^{\dagger}=H_{s_{x}, s_{y}, s_{z}}$ where $H_{s_{x}, s_{y}, s_{z}}=s_{x} \sigma_{x} \otimes \sigma_{x}+$ $s_{y} \sigma_{y} \otimes \sigma_{y}+s_{z} \sigma_{z} \otimes \sigma_{z}$. By computing the eigenvalues we see that $\left\|H_{s_{x}, s_{y}, s_{z}}\right\|=\sum_{\alpha}\left|s_{\alpha}\right|$. The simulation time overhead can not be more than the right hand side since each term $s_{\alpha} \sigma_{\alpha} \otimes \sigma_{\alpha}$ can be simulated with overhead $s_{\alpha}$.

Let $H$ contain local terms, i.e. $H=\sum_{\alpha} J_{\alpha \alpha} \sigma_{\alpha} \otimes \sigma_{\alpha}+1 \otimes a+b \otimes 1$. We can split $H=H^{\prime}+H^{\prime \prime}$ where $H^{\prime}$ is the non-local part and $H^{\prime \prime}$ the local one. By the Trotter formula we can simulate the parts independently. The simulation of $H^{\prime \prime}$ takes no time by assumption. It remains to show that $\left\|H^{\prime}\right\| \leq\|H\|$. We may assume that $H$ is invariant with respect to qubit permutation since $\left\|\frac{1}{2} H+\frac{1}{2} H_{e x}\right\| \leq\|H\|$ where $H_{e x}$ is the Hamiltonian obtained from $H$ by exchanging the qubits. By conjugation we can obtain a Hamiltonian of the form $H=H_{s_{x}, s_{y}, s_{z}}+s\left(1 \otimes \sigma_{x}+\sigma_{x} \otimes 1\right)$. By computing the eigenvalues we see that $\left\|H_{s_{x}, s_{y}, s_{z}}\right\| \leq\|H\|$.

\footnotetext{
${ }^{3}$ Here $\|$.$\| denotes the operator norm given by \|a\|:=\max _{x}\|a x\|$ where $x$ runs over the unit vectors of the corresponding Hilbert space.
} 
Corollary 2 Let $A=\left\{u_{k l}\right\}_{k, l}$ be a step of a quantum circuit and $\alpha$ its weighted depth. Then the zz-model can simulate the unitary implemented by A with overhead $\alpha$.

Proof: Let $M=\{(k, l)\}$ be the set of the pairs which the two-qubit gates act on. No two pairs in $M$ contain a common vertex and therefore we can simulate the Hamiltonian $H_{M}=\sum_{(k, l) \in M} \sigma_{z}^{k} \sigma_{z}^{l}$ with overhead 1. Let $H_{k l}$ be the Hamiltonian of minimal norm such that $u_{k l}=\exp \left(i H_{k l}\right)$ for every $(k, l) \in M$. Now we can simulate every $H_{k l}$ parallely with overhead less than $\left\|H_{k l}\right\|$ by conjugating $H_{M}$.

Our goal is to compare interactions with respect to the simulation complexity in our model given by the complete $z z$-interaction and the quantum circuit model. For doing so, we need some basic concepts of graph theory 10. A graph is an ordered pair $G=(V, E)$ with $V \subseteq\{1,2, \ldots, n\}$ and $E=\left\{e_{1}, e_{2}, \ldots, e_{m}\right\} \subseteq V \times V$. Elements of $V$ are called vertices. They label the qubits. Elements of $E$ are called edges. They label the pair-interactions between the qubits. An edge $e=(k, l)$ is an ordered pair of vertices $k$ and $l$ called the ends of $e$. We consider only undirected graphs with no loops. To have a unique representation we require that $k<l$. Two distinct edges are called adjacent if and only if they have a common end vertex. A subset $M$ of the edge set $E$ is called independent if no two edges of $M$ are adjacent in $G$. A graph $G$ is called complete if every pair of distinct vertices of $G$ are adjacent in $G$; such a graph is denoted by $K_{n}$. Rephrased in this language, our drift Hamiltonian is of the form

$$
H_{d}=\sum_{(k, l) \in E\left(K_{n}\right)} \sigma_{z}^{k} \sigma_{z}^{l}
$$

and is called in the following the complete $z z$-Hamiltonian.

Definition 5 Let $H$ be an arbitrary pair-interaction Hamiltonian. For every nonnegative real number $r$ we define the interaction graph $G_{r}$ as follows: Let the qubits $\{1, \ldots, n\}$ label the vertices and let the edges be all the pairs $(k, l)$ with the property $\left\|H_{k, l}\right\|>r$.

The chromatic index $\chi^{\prime}$ is the minimum number of colors permitting an edge-coloring such that no two adjacent edges receive the same color or equivalently a partition $E=M_{1} \cup M_{2} \cup \ldots \cup M_{\chi^{\prime}}$ into independent subsets of $E$. The following quantity turns out to be an upper bound on the overhead.

Definition 6 We define the weighted chromatic index of $H$

$$
\chi^{\prime}:=\int_{0}^{\infty} \chi_{r}^{\prime} d r
$$

where $\chi_{r}^{\prime}$ denotes the chromatic index of $G_{r}$.

In a former paper [11] we have introduced the weighted chromatic index as a complexity measure of the interaction. This point of view has been justified by two arguments, where the first one is an observation in [11]:

Theorem 2 The evolution generated by a pair-interaction Hamiltonian $H$ during the infinitesimal time period dt can be simulated by a parallelized 2-qubit gate network with weighted depth $\chi^{\prime} d t$ if $\chi^{\prime}$ is the weighted chromatic index of $H$. 
The second argument to consider chromatic index as a complexity measure for the interaction is only intuitive: in general, it should be easy to control interactions on disjoint qubit pairs, whereas one should expect that its unlikely that one can control simultaneously the interaction between qubit 1 and 2 and the interaction 1 and 3 at the same moment. This 'a priori'-assumption of [11] can be partly justified by the following corollary which is an easy conclusion of Corollary 2 and Theorem 2 .

Corollary 3 The time overhead for simulating the Hamiltonian $H$ in the zz-model is at most the weighted chromatic index of $H$.

The assumption that the drift Hamiltonian contains only pair-interaction of the form $\sigma_{z} \otimes \sigma_{z}$ can be dropped. Let $H=\sum_{\alpha, \beta} J_{\alpha \beta} \sigma_{\alpha} \otimes \sigma_{\beta}$ be an arbitrary pair-interaction. By conjugating $H$ with $\left\{I \otimes I, I \otimes \sigma_{z}, \sigma_{z} \otimes I, \sigma_{z} \otimes \sigma_{z}\right\}$ we obtain $J_{z z} \sigma_{z} \otimes \sigma_{z}$. This can be done with overhead 1 . The bounds of the corollary must be divided by the minimum $J_{z z}$ of all pair-interactions occurring in $H$.

\section{Applications}

The graph theoretical nature of our optimization problems becomes even stronger if we reduce our attention to one type of interactions, namely $z z$-interactions. Then the desired Hamiltonian is completely described by a weighted graph.

We consider the problem to simulate the time evolution

$$
H=\sum_{(k, l)} J_{k l} \sigma_{z}^{k} \sigma_{z}^{l}
$$

when the complete $z z$-Hamiltonian is present. We first show that in this case it is sufficient to use conjugation by $\sigma_{x}$ only. Let $H^{\prime}:=\sigma_{z} \otimes \sigma_{z}$. Note that $\left(\sigma_{x} \otimes I\right) H^{\prime}\left(\sigma_{x} \otimes\right.$ $I)=-H^{\prime}$ and $\left(\sigma_{x} \otimes \sigma_{x}\right) H^{\prime}\left(\sigma_{x} \otimes \sigma_{x}\right)=H^{\prime}$. In the following we denote conjugation by $\sigma_{x}$ by - and no conjugation by + . The Hamiltonian to be simulated contains only terms of the form $J_{k l ; z z} \sigma_{z}^{k} \sigma_{z}^{l}$ by assumption. If it is written as a convex combination of elements of $A d_{K}\left(H_{d}\right)$ it is sufficient to show that for each of these elements there is a procedure which cancels the terms $J_{k l ; \alpha \beta}$ for $(\alpha, \beta) \neq(z, z)$ without any effect on the $J_{k l, z z}$ terms. Therefore consider $\tilde{H}=k H_{d} k^{\dagger}$. Then we can also achieve the Hamiltonian $\tilde{H}_{z z}=\sum_{(k, l)} \tilde{J}_{k l ; z z} \sigma_{z}^{k} \sigma_{z}^{l}$ with overhead 1. For every qubit $i$ there is a $\tilde{J}_{i ; z}$ such that $\tilde{J}_{k l ; z z}=\tilde{J}_{k ; z} \tilde{J}_{l ; z}$ for all edges $(k, l)$. We express each $\tilde{J}_{i ; z}=c_{i}^{+}-c_{i}^{-}$with $0 \leq c_{k}^{+}, c_{k}^{-} \leq 1$ and $c_{k}^{+}+c_{k}^{-}=1$. Let $K=\left\{I, \sigma_{x}\right\} \otimes \ldots \otimes\left\{I, \sigma_{x}\right\}$. We conjugate the drift Hamiltonian by $u=u_{1} \otimes u_{2} \otimes \ldots \otimes u_{n} \in K$ for time $t(u)=\prod_{i=1}^{n} c_{i}(u)$ where $c_{i}(u)=c_{k}^{+}$if $u_{i}=I$ and $c_{i}(u)=c_{k}^{-}$if $u_{k}=\sigma_{x}$. We have

$$
\sum_{u \in K} t(u) u \tilde{H} u^{\dagger}=\tilde{H}_{z z}
$$

Since we restrict our attention to interactions with $z z$-terms only a shorter notation will be useful. To each edge $e=(k, l)$ of $G$, we associate a real number $w_{k l}$ called the weight of $e$. The resulting graph is called a weighted graph. Its adjacency matrix $J$ is the real symmetric matrix with zeros on the diagonal defined by

$$
J_{i i}:=0, \quad J_{k l}:=w_{k l} \text { and } J_{l k}:=w_{k l}
$$


for all edges $(k, l)$ of $G$. An unweighted graph can be considered as a weighted whose edges all have the weight 1 .

A (unweighted) graph is bipartite if its vertex set can be partitioned into two nonempty subsets $X$ and $Y$ such that each edge of $G$ has one end in $X$ and the other in $Y$. The pair $(X, Y)$ is called a bipartition of the bipartite graph. The complete bipartite graph with bipartition $(X, Y)$ is denoted by $G(X, Y)$.

A Seidel matrix defines a modified adjacency matrix $S=\left(s_{k l}\right)$ for (unweighted) graphs in the following way [12]:

$$
s_{k l}=\left\{\begin{aligned}
-1 & \text { if } k \text { and } l \text { are adjacent } k \neq l \\
1 & \text { if } k \text { and } l \text { are non-adjacent }
\end{aligned}\right.
$$

and $s_{k k}=0$. Obviously, $S=K-I-2 J$, where $K$ denotes a square matrix all of whose entries are equal to 1 and $J$ the adjacency matrix of $G$.

Theorem 3 (Optimal simulation) A graph $G$ can be simulated with overhead 1 if and only if it can be expressed as a convex combination

$$
J=\sum_{i} t_{i} S_{i}
$$

where the sum runs over the Seidel adjacency matrices of all complete bipartite graphs, i.e., over $2^{n-1}$ possible matrices.

Proof: By assigning to each vertex either + or - we have a bipartition of the vertex set: $X$ contains all vertices with + and $Y$ all vertices with - . The sign of the edge $(k, l)$ is - if and only if the edge has one end in $X$ and the other end in $Y$ and + otherwise. The edges with - define the complete bipartite graph $G(X, Y)$. We also include the case $X=\emptyset$ and $Y=V$ to cover the case when + is assigned to all knots. Therefore all we can achieve in the one step is $K-I-2 J(X, Y)$.

Corollary 4 (Lower bound) The absolute value of the smallest eigenvalue of the $J$-matrix is a lower bound on the simulation overhead.

We present now some upper bounds on the overhead. A graph $G=\left(V^{\prime}, E^{\prime}\right)$ is called a subgraph of $G^{\prime}$ if $V^{\prime} \subseteq V$ and $E^{\prime} \subseteq E$. A clique of $G$ is a complete subgraph of $G$. A clique of $G$ is called a maximal clique of $G$ if it is not properly contained in another clique of $G$. A clique partition $P$ of $G$ is a partition of $E(G)$ such that its classes induce maximal cliques of $G$. Given a set $C$ of $h$ colors, an $h$-coloring of $P$ in $G$ is a mapping from $P$ to $C$, such that cliques sharing a vertex have different colors. Let the clique coloring index $c(G)$ be the smallest $h$ such that there is a partition $P$ permitting an $h$-coloring [13]. We say the graph $G$ consists of independent cliques if $c(G)=1$.

Lemma 3 (Upper bound) Let $G$ be a graph consisting of independent cliques. We can simulate the Hamiltonian $H_{E}$ with overhead 1 which is optimal. 
Proof: Let $\omega \geq 2$ be the number of maximal cliques. We construct $\omega$ vectors $s_{i}$ of length $2^{\omega-1}$ as follows:

$$
\begin{aligned}
s_{1} & =(++++++++\cdots) \\
s_{2} & =(+-+-+-+-\cdots) \\
s_{3} & =(++--++--\cdots) \\
& \vdots \\
s_{\omega} & =(\underbrace{++\cdots+}_{2^{\omega-2}} \underbrace{--\cdots-\cdots}_{2^{\omega-2}})
\end{aligned}
$$

where + stands for 1 and - for -1 . The scalar products are $\left\langle s_{i}, s_{j}\right\rangle=2^{\omega-1} \delta_{i j}$. We partition the time interval into $2^{\omega-1}$ intervals of equal length. In the $m$ th interval we conjugate all qubits of the $i$ th clique by $\sigma_{x}$ if $s_{i, m}=-$ and do nothing otherwise. This is optimal since $q \leq-1$ where $q$ is the smallest eigenvalue of $G$.

The scheme used in the proof is time optimal. But the number of conjugations grows exponentially with the number of cliques $\omega$. It is possible to use the conjugations schemes based on Hadamard matrices [5]. There the number of conjugations grows only quadratically with $\omega$.

Corollary 5 (Upper bound) Let $G$ be an arbitrary graph. Then the Hamiltonian $H_{G}$ can be simulated with the overhead $c(G)$.

Note that if $M$ is an independent set than the graph $G=(V, M)$ consists of independent cliques. Therefore the chromatic index is an upper bound on clique index. However, this bounds is not always good. Consider e.g. the graph $G$ containing all edges that do not have 1 as end vertex. Then the chromatic index of $G$ is still high but the clique coloring index is only 1.

Since the optimal simulation of graph consisting of independent cliques has overhead 1 one might think that the clique index is the smallest overhead. But this is not so as shows the following example. Consider the star $G=(V, E)$ with $V=\{1, \ldots, 5\}$ and $E=\{(1,2),(1,3),(1,4),(1,5)\}$. The clique index of is 4 but the optimal simulation has overhead 2 only. The vectors can be chosen as $s_{1}=(++++), s_{2}=(-+++), s_{3}=$ $(+-++), s_{4}=(++-+), s_{5}=(+++-)$ and each of the four intervals has length $1 / 2$. This is optimal since the smallest eigenvalue of the adjacency matrix of $G$ is -2 .

\section{Quasi-order of Hamiltonians}

Let $H$ and $\tilde{H}$ be arbitrary pair-interaction Hamiltonians. We investigate the question whether $\tilde{H}$ can be simulated by $H$ with overhead $\mu$. Note that this defines a quasiorder of the pair-interaction Hamiltonians for $\mu=1$. A partial characterization of the quasi-order is expressed in terms of majorization of the spectra of the corresponding matrices $J$ and $\tilde{J}$. Similar methods have been used to derive conditions for a class of entanglement transformations and to characterize mixing and measurement in quantum mechanics [6, 7].

Suppose that $x=\left(x_{1}, \ldots, x_{d}\right)$ and $y=\left(y_{1}, \ldots, y_{d}\right)$ are two dimensional real vectors. We introduce the notation $\downarrow$ to denote the components of a vector rearranged into nonincreasing order, so $x^{\downarrow}=\left(x_{1}^{\downarrow}, \ldots, x_{d}^{\downarrow}\right)$, where $\left(x_{1}^{\downarrow} \geq x_{2}^{\downarrow} \geq \ldots \geq x_{d}^{\downarrow}\right)$. We say that $x$ is 
majorized by $y$ and write $x \prec y$, if

$$
\sum_{j=1}^{k} x_{j}^{\downarrow} \leq \sum_{j=1}^{k} y_{j}^{\downarrow}
$$

for $k=1, \ldots, d-1$, and with equality when $k=d[8]$.

Let $\operatorname{Spec}(X)$ denote the spectrum of the hermitian matrix $X$, i.e. the vector of eigenvalues, and $\lambda(X)$ denote the vector of components of $\operatorname{Spec}(X)$ arranged so they appear in non-increasing order. Ky Fan's maximum principle [7] states that for any Hermitian matrix $A$, the sum of the $k$ largest eigenvalues of $A$ is the maximum value $\operatorname{tr}(A P)$, where the maximum is taken over all $k$-dimensional projections $P$,

$$
\sum_{j=1}^{k} \lambda_{j}(A)=\max _{P} \operatorname{tr}(A P) .
$$

It gives rise to a useful constraint on the eigenvalues of a sum of two Hermitian matrices $C:=A+B$, that $\lambda(C) \prec \lambda(A)+\lambda(B)$. Choose a $k$-dimensional projection $P$ such that

$$
\sum_{j=1}^{k} \lambda_{j}(C)=\operatorname{tr}(C P)=\operatorname{tr}(A P)+\operatorname{tr}(B P) \leq \sum_{j=1}^{k} \lambda_{j}(A)+\sum_{j=1}^{k} \lambda_{j}(B) .
$$

This permits us to derive a lower bound on the simulation overhead.

Lemma 4 (Majorization) Let $H$ and $\tilde{H}$ be arbitrary pair-interaction Hamiltonians. A necessary condition that $\tilde{H}$ can be simulated with overhead $\mu$ by $H$ is that $\operatorname{Spec}(\tilde{J}) \prec$ $\mu \operatorname{Spec}(J)$.

Proof: By representing the Hamiltonians by their $J$-matrices we see that $\tilde{H}$ can be simulated with overhead $\mu$ if and only if there is a sequence of orthogonal matrices $U_{j}=U_{j 1} \oplus \ldots U_{j n} \in S O(3) \oplus \ldots \oplus S O(3)$ and $\mu_{j}>0$ with $\sum_{j} \mu_{j}=\mu$ such that

$$
\tilde{J}=\sum_{j} \mu_{j} U_{j} J U_{j}^{T}
$$

The proof now follows from the inequality (11).

We consider now the problem to reverse the time evolution $\exp \left(i H_{d} t\right)$, i.e. what is the overhead of simulating $-H_{d}$ when $H_{d}$ is present.

Lemma 5 (Lower bound on inverting) Let $r$ be the greatest eigenvalue and $q$ the smallest eigenvalue of $J$. Then $\mu \geq \frac{r}{-q}$ is a lower bound on the overhead for simulating $-H_{d}$ by $H_{d}$.

Proof: This is a direct consequence of the Weyl inequality (see [8], Theorem III.2) $\lambda_{d}(A+B) \geq \lambda_{d}(A)+\lambda(B)$ for the sum of two Hermitian matrices where $\lambda_{d}$ denotes the smallest eigenvalue.

Let $G$ be a connected graph and $H_{d}=\sum_{(k, l) \in E(G)} \sigma_{z}^{k} \sigma_{z}^{l}$. If $G$ is not connected then the components can be treated independently. For the spectrum the following statements hold (see [12], Theorem 0.13):

$$
1 \leq r \leq n-1, \quad-r \leq q \leq-1 .
$$


It is interesting to note that this gives a tight bound for simulating $-H_{d}$ when $G=K_{n}$ since for a complete graph we have $r=(n-1)$ and $q=-1$. An upper bound is the (weighted) chromatic index $\chi^{\prime}\left(K_{n}\right)$ which is either $n$ (if $n$ is even) or $n-1$ (otherwise). This simple example shows that the inverse of the natural time evolution may have a relatively high complexity.

Lemma 6 Let the drift Hamiltonian $H_{d}$ be an arbitrary pair-interaction Hamiltonian. If the interaction graph $G_{0}\left(H_{d}\right)$ is bipartite then we can invert the time evolution with overhead of less than 3.

Proof: Let $S=\left\{\sigma_{x}, \sigma_{y}, \sigma_{z}\right\}$. We have $\sum_{u \in S} u a u^{\dagger}=-a$ for all $a \in \mathfrak{s u}(2)$. Let $X, Y$ be the bipartition of $G_{0}\left(H_{d}\right)$. By conjugating all qubits in $X$ with elements of $S$ we obtain $-H_{d}$.

If $H$ contains only $\sigma_{z} \otimes \sigma_{z}$ then the overhead is 1 . This is optimal since we have $\mu \geq 1$.

\section{References}

[1] M. A. Nielsen and I. L. Chuang, Quantum Computation and Quantum Information, Cambrige, 2000.

[2] N. Duffield, and R. Werner, Local dynamics of mean-field quantum systems, Helv. Phys. Acta, Vol. 95, pp. 1016-1054, 1992.

[3] N. Khaneja, R. Brockett, and S. J. Glaser, Time optimal control in spin systems, Phys. Rev. A, Vol. 63, pp. 032308 1-13, 2001.

[4] J. L. Dodd, M. A. Nielsen, M. J. Bremner, and R. T.Thew, Universal quantum computation and simulation using any entangling Hamiltonian and local unitaries, quant-ph/0106064 v2.

[5] D. W. Leung, I. L. Chuang, F. Yamaguchi, and Y. Yamamoto, Efficient implementation of coupled logic gates for quantum computing, Phys. Rev. A, Vol. 61, pp. 042310-0-7, 2000.

[6] M. A. Nielsen, Conditions for a class of entanglement transformations, Phys. Rev. Lett., Vol. 83, pp. 436-439, 1999.

[7] M. A. Nielsen, Characterizing mixing and measurements in quantum mechanics, quant-ph/0008073, 2000.

[8] R. Bhatia, Matrix Analysis, Graduate texts in mathematics; 169, Springer, 1996.

[9] R. A. Horn, and C. R. Johnson, Matrix Analysis, Cambridge University Press, 1985.

[10] B. Bollobás, Modern Graph Theory, Springer Verlag, 1998.

[11] D. Janzing and Th. Beth, A complexity measure for continuous time quantum algorithms, quant-ph/0009094, 2000, to appear in Phys. Rev. A.

[12] D. M. Cvetković, M. Doob, and H. Sachs, Spectra of Graphs: theory and applications, 3rd edition, Johann Ambrosius Barth Verlag, 1995.

[13] W. D. Wallis, Guo-Hui Zhang, On the partition and coloring of a graph by cliques, Discrete Math. 120., No. 1-3, pp. 191-203, 1993. 